\title{
A 1-bp deletion in bovine $Q R I C H 2$ causes low sperm count and immotile sperm with multiple morphological abnormalities
}

\author{
Maya Hiltpold ${ }^{1 *}$ (D, Fredi Janett ${ }^{2}$, Xena Marie Mapel ${ }^{1}$, Naveen Kumar Kadri ${ }^{1}$, Zih-Hua Fang ${ }^{1,6}$, \\ Hermann Schwarzenbacher ${ }^{3}$, Franz R. Seefried ${ }^{4}$, Mirjam Spengeler ${ }^{4}$, Ulrich Witschi ${ }^{5}$ and Hubert Pausch ${ }^{1}$
}

\begin{abstract}
Background: Semen quality and insemination success are monitored in artificial insemination bulls to ensure high male fertility rates. Only ejaculates that fulfill minimum quality requirements are processed and eventually used for artificial inseminations. We examined 70,990 ejaculates from 1343 Brown Swiss bulls to identify bulls from which all ejaculates were rejected due to low semen quality. This procedure identified a bull that produced 12 ejaculates with an aberrantly small number of sperm $\left(0.2 \pm 0.2 \times 10^{9}\right.$ sperm per $\left.\mathrm{mL}\right)$ which were mostly immotile due to multiple morphological abnormalities.
\end{abstract}

Results: The genome of this bull was sequenced at a $12 \times$ coverage to investigate a possible genetic cause. Comparing the sequence variant genotypes of this bull with those from 397 fertile bulls revealed a 1-bp deletion in the coding sequence of the QRICH2 gene which encodes the glutamine rich 2 protein, as a compelling candidate causal variant. This 1-bp deletion causes a frameshift in translation and a premature termination codon (ENSBTAP00000018337.1:p.Cys1644AlafsTer52). The analysis of testis transcriptomes from 76 bulls showed that the transcript with the premature termination codon is subject to nonsense-mediated mRNA decay. The 1-bp deletion resides in a 675-kb haplotype that includes 181 single nucleotide polymorphisms (SNPs) from the Illumina BovineHD Bead chip. This haplotype segregates at a frequency of $5 \%$ in the Brown Swiss cattle population. Our analysis also identified another bull that carried the 1-bp deletion in the homozygous state. Semen analyses from the second bull confirmed low sperm concentration and immotile sperm with multiple morphological abnormalities that primarily affect the sperm flagellum and, to a lesser extent, the sperm head.

Conclusions: A recessive loss-of-function allele of the bovine $Q R I C H 2$ gene likely causes low sperm concentration and immotile sperm with multiple morphological abnormalities. Routine sperm analyses unambiguously identify homozygous bulls for this allele. A direct gene test can be implemented to monitor the frequency of the undesired allele in cattle populations.

*Correspondence: maya.hiltpold@usys.ethz.ch

1 Animal Genomics, Institute of Agricultural Sciences, ETH Zürich, Universitätstrasse 2, 8092 Zürich, Switzerland

Full list of author information is available at the end of the article

\section{Background}

Semen quality and male fertility are complex traits with low to moderate heritabilities [1]. These traits are routinely monitored in thousands of bulls as a service from the semen collection centers to the cattle breeding industry to ensure high success rates in artificial insemination. The availability of large cohorts with repeated semen quality analyses facilitates the separation between original author(s) and the source, provide a link to the Creative Commons licence, and indicate if changes were made. The images or other third party material in this article are included in the article's Creative Commons licence, unless indicated otherwise in a credit line to the material. If material is not included in the article's Creative Commons licence and your intended use is not permitted by statutory regulation or exceeds the permitted use, you will need to obtain permission directly from the copyright holder. To view a copy of this licence, visit http://creativecommons.org/licenses/by/4.0/. The Creative Commons Public Domain Dedication waiver (http://creativeco mmons.org/publicdomain/zero/1.0/) applies to the data made available in this article, unless otherwise stated in a credit line to the data. 
genetic and environmental factors and the differentiation between female and male factors that contribute to variation in reproduction [2]. Such phenotypes are ideally suited to characterize the genetic architecture of male fertility [3].

Genome-wide association testing revealed quantitative trait loci (QTL) for semen quality and male fertility in various breeds of cattle, many of them expressing non-additive effects [4-6]. The routine evaluation and recording of tens of thousands of ejaculates and millions of artificial inseminations also occasionally detect bulls with aberrant semen quality or strikingly low insemination success rates. Although diseases and environmental exposures can compromise male fertility [7], a drastically reduced semen quality in individual bulls often results from monogenic disorders [8-11]. Genomewide case-control association testing helps to pinpoint genetic variants associated with such conditions. In this approach, genotypes at genome-wide markers are compared between affected bulls and an unaffected control group. Variants at which the genotypes differ between both groups are candidate causal variants that are subsequently subjected to further functional investigations.

Impaired semen quality and low fertility in healthy bulls can be caused by deleterious alleles at genes that show testis-biased or testis-specific expression [8-10]. Such alleles are spread disproportionately by females because they are typically not impacted by defective genes that are primarily expressed in the male reproductive system. Semen quality and male fertility are only evaluated in breeding bulls. Thus, recessive variants that compromise male reproduction can remain undetected in cattle populations for a long period of time or reach a high allele frequency without being detected [8].

Here we report a 1-bp deletion inducing a frameshift in the bovine QRICH2 gene that, in the homozygous state, likely causes male infertility due to immotile sperm with multiple morphological abnormalities. Using historic and current semen quality data from two Brown Swiss bulls, we show that this mutation remained undetected although phenotypic consequences started to be observed almost two decades ago. Our findings offer the opportunity to implement direct gene testing to monitor the frequency of this undesired allele in cattle populations.

\section{Methods}

\section{Animals and phenotypes}

We assessed records for 70,990 ejaculates that were collected from 1343 Brown Swiss bulls between January 2000 and March 2018. All ejaculates were examined by laboratory technicians employed by Swissgenetics immediately after semen collection as part of routine quality assurance during semen processing. The parameters recorded were semen volume $(\mathrm{mL})$, sperm concentration (million sperm per $\mathrm{mL}$ ) that was quantified using photometric analysis, and the percentage of sperm with forward motility. The proportion of sperm with head and tail anomalies was quantified for each ejaculate with scores ranging from 0 to 3 (0: no or very few anomalies, 1 : less than $10 \%$ sperm with anomalies, 2 : between 10 and $30 \%$ sperm with anomalies, 3 : more than $30 \%$ sperm with anomalies). Subsequently, we considered 35,785 ejaculates that were collected from 1258 bulls aged between 330 and 550 days to identify those for which all ejaculates were discarded due to insufficient semen quality. Ejaculates that contained less than 300 million sperm per $\mathrm{mL}$, less than $70 \%$ motile sperm, more than $10 \%$ sperm with head and tail abnormalities, or with a volume smaller than $1 \mathrm{~mL}$ were deemed to be unsuitable for artificial inseminations.

\section{Whole-genome sequencing and sequence variant genotyping}

We extracted DNA from hair roots of a Brown Swiss bull that produced ejaculates with a low sperm count and immotile sperm with multiple morphological abnormalities (since the sequence read archive accession number is SAMEA6272098, this bull is hereafter referred to as SAMEA6272098). For whole-genome sequencing, an Illumina TruSeq DNA PCR-free paired-end library with a 400-bp insert size was sequenced on an Illumina NovaSeq6000 instrument. We used the fastp software [12] to remove adapter sequences, poly- $G$ tails and reads that had a phred-scaled quality score less than 15 for more than $15 \%$ of the bases. Following quality control, we aligned 153,618,844 read pairs to the ARS-UCD1.2 assembly of the bovine genome using the mem-algorithm of the BWA software [13] with option -M to mark shorter split hits as secondary alignments and default settings for all other parameters. We marked duplicates using the Picard tools software suite (https://github.com/broad institute/picard). Alignments were sorted by coordinates using the Sambamba tool [14]. Sequencing depth was calculated using the mosdepth software (version 0.2.2, [15]) by considering only the reads with a mapping quality higher than 10 .

The SAMEA6272098 bull and 521 cattle from various breeds (14 Hereford, 1 Nelore, 33 Grauvieh, 50 Fleckvieh, 2 Nordic Red Cattle, 47 Holstein, 243 Brown Swiss, 128 Original Braunvieh, and 3 Wagyu) were genotyped for sequence variants (single nucleotide polymorphisms (SNPs) and insertion-deletion mutations (indels)) using a multi-sample variant discovery and genotyping approach implemented with the HaplotypeCaller, GenomicsDBImport and GenotypeGVCFs modules from the Genome 
Analysis Toolkit (GATK, [16]). Subsequently, we applied best practice guidelines of the GATK for variant filtration and imputed missing genotypes using Beagle [17]. The reference-guided variant genotyping workflow applied here is described in detail in Crysnanto et al. [18]. Functional consequences of 41,659,308 autosomal variants were predicted according to the Ensembl (version 104) and Refseq (version 106) annotations of the bovine genome using the Variant Effect Predictor software [19] from Ensembl along with the SpliceRegion.pm plugin (https://github.com/Ensembl/VEP_plugins/blob/release/ 101/SpliceRegion.pm).

\section{Identification of candidate causal variants}

We considered the whole sequences of 397 fertile bulls as controls to identify sequence variant genotypes associated with the sperm disorder of the SAMEA6272098 bull (see Additional file 1: Table S1). These 397 bulls were either key ancestor animals or artificial insemination bulls or both and as such were assumed to be fertile, although sperm quality was not examined for all of them. Assuming recessive inheritance of a deleterious allele, we screened the sequence variant genotypes of the SAMEA6272098 genome for homozygous sites that were never found in the homozygous state in fertile bulls. These variants were subsequently filtered to retain those with a predicted deleterious impact on a protein.

\section{Identification of haplotype carriers in the population}

Imputed high-density genotypes of 33,045 cattle were used to assign the 1-bp deletion to a haplotype. Briefly, these 33,045 cattle were genotyped using different Illumina SNP microarrays [4]. Following quality control, the genotypes were imputed to a density of 683,903 SNPs with Beagle [20] using a reference panel of 1166 cattle that had BovineHD genotypes. We retained genotypes from 25,243 Brown Swiss and 5228 Original Braunvieh animals from the Swiss herdbook populations. A subset of the genotyped animals $(\mathrm{N}=285)$ also had wholegenome sequence-derived genotypes.

We inspected the phased (and partially imputed) BovineHD genotypes of 285 animals which also had the 1-bp deletion genotyped using whole-genome sequencing data. Specifically, we searched for a haplotype that extends on either side of the BTA19:55436705TC $>$ T position of the deletion (BTA19 for Bos taurus chromosome 19) and that was shared in the heterozygous state by all sequenced animals carrying the 1-bp deletion. The genotypes and haplotypes of the 285 animals are in Additional file 1: Table S1. We used the alleles of the longest shared haplotype to identify heterozygous and homozygous haplotype carriers among 30,471 genotyped Brown Swiss and Original Braunvieh cattle. Alleles and positions of markers encompassed by the haplotype (see Additional file 2: Table S2) were also provided to Brown Swiss breeding associations and genetic evaluation centers to screen their genomic prediction reference populations and selection candidates for homozygous haplotype carriers.

\section{Whole transcriptome sequencing and read alignment}

We used DNA and RNA sequencing data from a previously established expression QTL (eQTL) cohort to investigate transcript abundance and functional consequences caused by the 1-bp deletion. The eQTL cohort consisted of 76 mature bulls from which the testes were sampled at a commercial slaughterhouse after regular slaughter. Total RNA and DNA (see Additional file 3: Table S3) were extracted from testis tissue and prepared for sequencing as described earlier [21].

DNA samples were sequenced on an Illumina NovaSeq6000 sequencer using 150-bp paired-end libraries. Quality control (removal of adapter sequences and low-quality bases, and trimming of poly-G tails) of the raw sequencing data was carried out using the fastp software [12] with default parameters. Following quality control, between 70,493,763 and 307,416,205 read pairs per sample were aligned to the ARS-UCD1.2 version of the bovine reference genome [22] using the mem-algorithm of the BWA software (see above). Average coverage of the 76 DNA samples estimated using the mosdepth software (see above) ranged from 6.3 to 27.6 with a mean value of $12.6 \pm 4.2$. Sequence variant genotypes were called and filtered using the HaplotypeCaller, GenomicsDBImport, GenotypeGVCFs and VariantFiltration modules from the GATK as described above.

Total RNA sequencing libraries $(2 \times 150 \mathrm{bp})$ were prepared using the Illumina TruSeq Stranded Total RNA sequencing kit and sequenced on an Illumina NovaSeq6000 sequencer. Quality control (removal of adapter sequences and low-quality bases, and trimming of poly-A and poly-G tails) of the raw sequencing data was carried out using the fastp software [12]. Following quality control, between 191,160,837 and 386,773,085 filtered reads per sample (mean: $283,587,831 \pm 43,284,185$ ) were aligned to the ARS-UCD1.2 reference sequence and the Ensembl gene annotation (release 104) using STAR (version 2.7.9a) [23] with options -twopassMode Basic, -sjdbOverhang 100, -outFilterMismatchNmax 3, and -outSAMmapqUnique 60.

\section{Bioinformatic analysis}

The structure of the bovine QRICH2 gene (ENSBTAG00000030173, Gene-ID 530282) was analysed using data from Ensembl (version 104) and Refseq (version 106). Transcript abundance (in transcripts per million, 
TPM) was quantified for the 76 bulls of the eQTL cohort using kallisto [24] and aggregated to the gene level using the $\mathrm{R}$ package tximport [25]. Exon abundance was quantified using QTLtools [26]. Exon-exon junctions were visualized using the ggsashimi $R$ package [27]. Read coverage as well as reference and alternative allele support at the 1-bp deletion were inspected using the Integrative Genomics Viewer (IGV, [28]). A binomial test was carried out with the $\mathrm{R}$ function binom.test() to examine if the alternate allele ratio in six heterozygous bulls was significantly different from 0.5 .

\section{Genotyping of the 1-bp deletion}

Polymerase chain reaction (PCR) and Sanger sequencing were used to validate the 1-bp deletion. PCR products from genomic DNA were amplified using the following primers: 5'-CATCGAGAAGGTGCAGATCC-3' (forward) and 5'-CTGCCC ACCGTTTGTAGC-3' (reverse) with a standard $50 \mu \mathrm{L}$ PCR reaction mix (100 ng genomic DNA and final concentrations of $1 \times$ PCR reaction buffer, $200 \mu \mathrm{M}$ dNTP mixture, $0.2 \mu \mathrm{M}$ each primer and 0.05 units $/ \mu \mathrm{L}$ JumpStart Taq Polymerase (Sigma)). The PCR amplification program consisted of an initial denaturation step at $94{ }^{\circ} \mathrm{C}$ for $30 \mathrm{~s}$ followed by 30 cycles of $94{ }^{\circ} \mathrm{C}$ for $10 \mathrm{~s}, 55^{\circ} \mathrm{C}$ for $1 \mathrm{~min}$, and $72{ }^{\circ} \mathrm{C}$ for $30 \mathrm{~s}$ with a final extension step at $72{ }^{\circ} \mathrm{C}$ for $1 \mathrm{~min}$. All PCR products were purified with the GenElute PCR Clean-Up Kit (Sigma) and sent for Sanger sequencing (Microsynth, Switzerland). Sequencing results were analysed using the CLC Genomics Workbench software (Qiagen).

\section{Analysis of the semen from a bull homozygous for the 1-bp deletion}

Ejaculates from a bull homozygous for the 1-bp deletion were collected at 14, 16, 17 and 20 months of age using an artificial vagina. Sperm concentration, total sperm count, and sperm motility were determined with an IVOS II CASA system (Hamilton Thorne Inc., Beverly, USA) using Leja 2-chamber slides (Leja, NieuwVennep, the Netherlands). Semen was fixed in buffered formol saline solution $\left(\mathrm{Na}_{2} \mathrm{HPO}_{4} 4.93 \mathrm{~g}, \mathrm{KH}_{2} \mathrm{PO}_{4} 2.54 \mathrm{~g}\right.$, $38 \%$ formaldehyde $125 \mathrm{~mL}, \mathrm{NaCl} 5.41 \mathrm{~g}$, distilled water qs $1000 \mathrm{~mL}$ ) and smears were prepared according to Hancock [29] for morphological examination. At least 200 sperm were subsequently evaluated by phase contrast microscopy using oil immersion (Olympus BX50, UplanF1 100×/1.30, Olympus, Wallisellen, Switzerland). Morphologically abnormal sperm were assigned to defect categories according to Blom [30].

Sperm viability was assessed using the eosin-nigrosin staining method [31]. At least 200 sperm were evaluated on stained slides under oil immersion using a light microscope (Olympus BX50, UplanF1 100×/1.30, Olympus, Wallisellen).

The bull that was homozygous for the 1-bp deletion was slaughtered at 21 months at a regular slaughterhouse. Testis tissue was preserved in formalin, embedded in paraffin and subsequently stained with hematoxylin-eosin for histological evaluation.

\section{Transmission electron microscopy}

Sperm were prepared for transmission electron microscopy (TEM) from fresh semen diluted in OptiXcell (IMV technologies, L'Aîgle, France) and stored at $5{ }^{\circ} \mathrm{C}$ overnight. Semen samples were washed twice by dilution in phosphate buffered saline (PBS) and subsequent centrifugation at $300 \mathrm{~g}$ for $10 \mathrm{~min}$. After removing the supernatant, the pellet was fixed with an equal volume of 6\% glutaraldehyde in PBS, resuspended gently, and centrifuged at $6000 \mathrm{~g}$. After removing the supernatant, the sperm were fixed for a second time with $3 \%$ glutaraldehyde in PBS, and finally pelleted at $6000 \mathrm{~g}$. Pellets were washed three times in PBS, post-fixed in $1 \%$ osmium tetroxide, washed in double distilled water, stained in $1 \%$ uranyl acetate, dehydrated in a graded series of ethanol $(25,50,75,90$, and $100 \%)$, and embedded in epoxy resin through increasing concentrations $(25,50,75$, and $100 \%)$ using PELCO BioWave Pro + tissue processor (Ted Pella, USA), and then cured at $60{ }^{\circ} \mathrm{C}$ for 3 days. Embedded blocks were sectioned using the Leica FC6 microtome and a DIATOME diamond knife at a $45^{\circ}$ angle into $60-\mathrm{nm}$ sections and mounted on Quantifoil copper grids with formvar carbon films. Sections were post-stained with $2 \%$ uranyl acetate followed by lead citrate. Grids were imaged using a FEI Morgagni 268 electron microscope operated at $100 \mathrm{kV}$ at 8.9 to $56 \mathrm{k}$ magnification.

\section{Scanning electron microscopy}

Sperm were prepared for scanning electron microscopy (SEM) within $30 \mathrm{~min}$ after semen collection. A thin layer of native semen was spread on carbon coated coverslips with the side of a pipet tip. The coverslips were immersed in 3\% glutaraldehyde in PBS solution and kept on ice. After fixation overnight at $4{ }^{\circ} \mathrm{C}$, fresh fixative was added and the sample was put in a PELCO BioWave Pro + microwave system (Ted Pella, USA). Following the microwave-assisted fixation and dehydration procedure, another fixation on ice was performed. After washing in PBS, samples were post-fixed in $1 \% \mathrm{OsO}_{4}$ in double distilled water, washed again and dehydrated in a graded series of ethanol $(50,75,90,98$, and three times $100 \%$ ) on ice followed by critical point drying out of dry ethanol (CPD 931, Tousimis, USA). The resulting samples were mounted on SEM aluminium stubs, fixed on conductive carbon tape and then sputter-coated with 
$4 \mathrm{~nm}$ of platinum/palladium (CCU-10, Safematic, $\mathrm{CH}$ ). Acquisition of images was performed using In-lens and Everhart-Thornley secondary electron (SE) signals at a working distance of $4 \mathrm{~mm}$ with a scanning electron microscope (Merlin FE-SEM, Zeiss, DE), operated at an accelerating voltage of $1.5 \mathrm{kV}$.

\section{Results}

\section{Identification of a Brown Swiss bull with poor semen quality}

We examined historic semen quality records from a semen collection centre in Switzerland as part of our ongoing efforts to investigate inherited variation in male reproduction in Brown Swiss bulls [1, 4, 32]. Among 1343 Brown Swiss bulls that produced 70,990 ejaculates, we identified seven bulls from which all ejaculates (between 5 and 28 per bull) were rejected due to immotile (asthenozoospermia), morphologically abnormal (teratozoospermia), low concentration (oligozoospermia), or absence of (azoospermia) sperm, or a combination of these anomalies. Because genetic material was not available for all these affected bulls, we focused on one of these bulls (born in 2003) from which preserved hair roots provided a source of DNA for genetic investigations.

Twelve ejaculates from this bull were collected between 15 and 18 months of age. The ejaculates had larger volumes $(6.6 \pm 1.5$ vs. $4.7 \pm 2.0 \mathrm{~mL})$ and lower sperm concentrations $\left(0.2 \pm 0.2\right.$ vs. $1.3 \pm 0.5 \times 10^{9}$ sperm cells per $\mathrm{mL}$ ) than observed in other Brown Swiss bulls of the same age. Almost all sperm (99\%) were immotile and had head and tail abnormalities. The proportion of viable sperm was not recorded. Such findings are commonly referred to as oligoasthenoteratozoospermia.

\section{Candidate causal variants}

The genome of the bull that produced ejaculates of insufficient quality was sequenced at 11.8-fold coverage using paired-end libraries (sequencing read archive accession: SAMEA6272098, thus this bull is referred to by this accession number). Its sequence variant genotypes were compared to those of 397 fertile artificial insemination bulls from various breeds that had an average sequencing coverage of $10.0 \pm 5.7$ fold (between 3.1- and 56.8-fold).
We hypothesized that the drastically reduced semen quality of SAMEA6272098 was due to a recessively inherited deleterious allele. From a catalogue of 41,659,308 autosomal sequence variants, we retained 1655 that were homozygous in SAMEA6272098 but not homozygous in the fertile control bulls (see Additional file 4: Table S4). Only three compatible variants were predicted to be deleterious to protein function: a nonsense variant in $M L N R$ that encodes the motilin receptor, a frameshift variant in QRICH2 that encodes the glutamine-rich protein 2, and a nonsense variant in ENSBTAG00000023270 that encodes an uncharacterized protein (Table 1). Another nine (Ensembl) and ten (Refseq) compatible variants were predicted to have moderate impacts on the corresponding proteins.

In the testis transcriptomes from 76 mature bulls, MLNR and ENSBTAG00000023270 were expressed with less than 0.1 transcripts per million (TPM), whereas QRICH2 was transcribed in high abundance (31.43 TPM). Moreover, QRICH2 shows an extremely testisbiased expression in mammals (e.g., https://gtexportal. org/home/, http://cattlegeneatlas.roslin.ed.ac.uk/) [33]. Pathogenic alleles of the human and mouse QRICH2 gene cause sperm with multiple morphological abnormalities of the flagella [34-36]. Thus, we considered QRICH2 as a compelling functional candidate gene for the sperm defect of SAMEA6272098 and pursued our analyses on this gene.

\section{A 1-bp deletion in the coding sequence of $Q R I C H 2$ is associated with aberrant semen quality}

The candidate causal variant that underlies the sperm defect is a 1-bp deletion in exon 16 of QRICH2 (at BTA19:55436705TC $>$ T, ENSBTAT00000018337.1:c .4929del). This deletion of a cytosine residue is predicted to alter the reading frame from amino acid 1644 onwards, which results in a premature termination codon (ENSBTAP00000018337.1:p.Cys1644AlafsTer52). This leads to a truncated protein that lacks 131 amino acids (7\%) from the C-terminal region unless the transcript is subjected to nonsense-mediated mRNA decay.

The pedigree-derived coefficient of inbreeding of the SAMEA6272098 bull is 7.61\%. Due to this relatively high

Table 1 High impact variants compatible with recessive inheritance

\begin{tabular}{|c|c|c|c|c|c|c|c|}
\hline Chr & Pos & Ref & Alt & Gene & $\begin{array}{l}\text { Transcript abundance in } \\
\text { testis (TPM) }\end{array}$ & Impact on protein & $\begin{array}{l}\text { Ensembl/ } \\
\text { refseq/ } \\
\text { both }\end{array}$ \\
\hline 12 & $18,859,636$ & $C$ & A & MLNR & $0.07 \pm 0.04$ & Cys587Ter & Both \\
\hline 19 & $55,436,705$ & TC & T & $\mathrm{QRICH} 2$ & $31.43 \pm 7.35$ & Cys1644AlafsTer52 & Both \\
\hline 24 & $10,181,719$ & G & $A$ & ENSBTAG00000023270 & $0.04 \pm 0.03$ & Trp248Ter & Ensembl \\
\hline
\end{tabular}

Chr: chromosome number; Pos: position of the variant on the genome; Ref: reference allele; Alt: alternate allele 
inbreeding, several extended segments of autozygosity are present in the genome of SAMEA6272098. The 1-bp deletion also resides within an approximately $3.625-\mathrm{Mb}$ segment (between 55 and $58.625 \mathrm{Mb}$ ) of high homozygosity (Fig. 1). This pattern suggests that the 1-bp deletion resides in a haplotype that SAMEA6272098 inherited from an ancestor that is present both in its maternal and paternal ancestry.

Among 244 Brown Swiss and 124 Original Braunvieh cattle, which we had sequenced for previous projects and which are representative animals from both populations, we detected the 1-bp deletion in the heterozygous state in 25 Brown Swiss and 3 Original Braunvieh individuals, corresponding to an allele frequency of 5.1 and $1.2 \%$, respectively. The heterozygous Original Braunvieh bulls were from Germany and we cannot rule out the possibility that the variant was recently introgressed through cross-breeding with Brown Swiss ancestors. The mutation did not segregate among 110 sequenced Original Braunvieh cattle from the Swiss herdbook population. We did not detect the mutation in breeds other than Brown Swiss and Original Braunvieh.

In a catalogue of variants that was established by the 1000 Bull Genomes Project consortium for 3934 cattle from various breeds, the 1-bp deletion was discovered in 16 animals from the Brown Swiss and one animal from the Nordic Red Dairy cattle breeds. The carrier animal from the Nordic Red Dairy cattle breed had an estimated
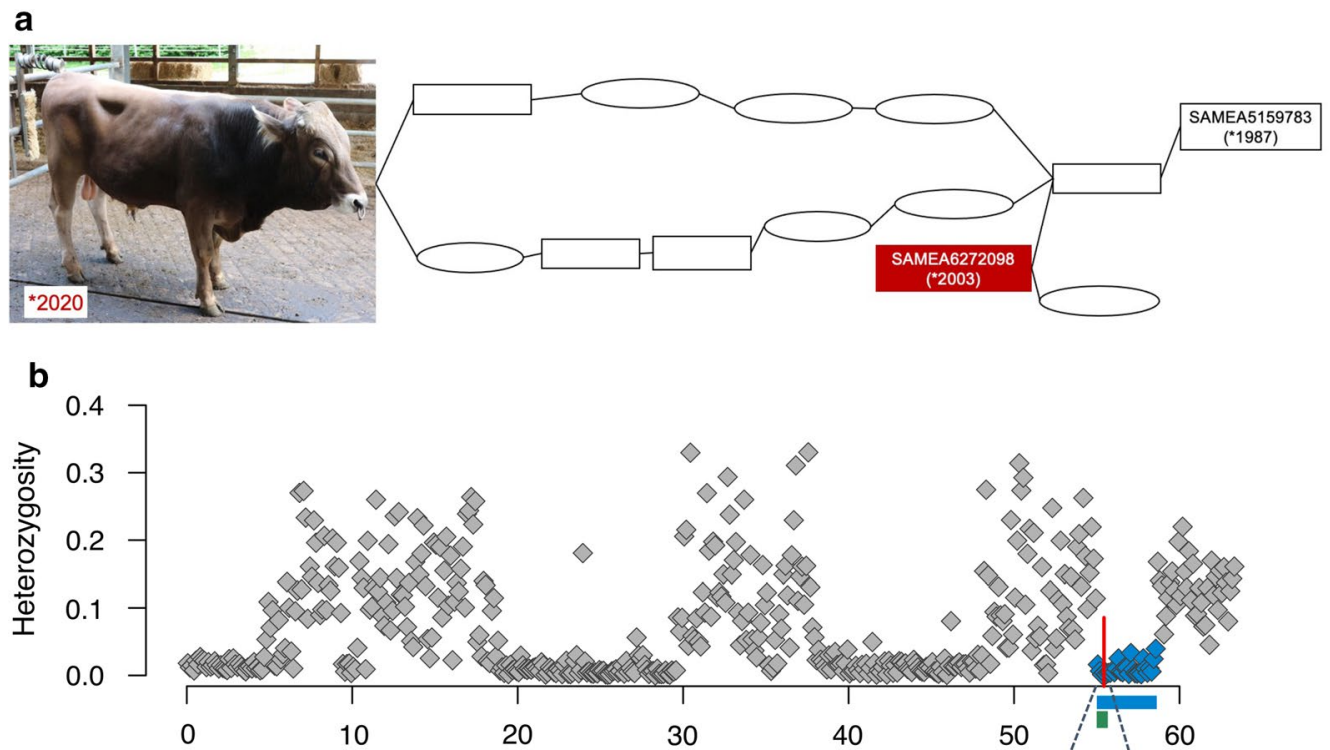

Position on chromosome $19(\mathrm{Mb})$

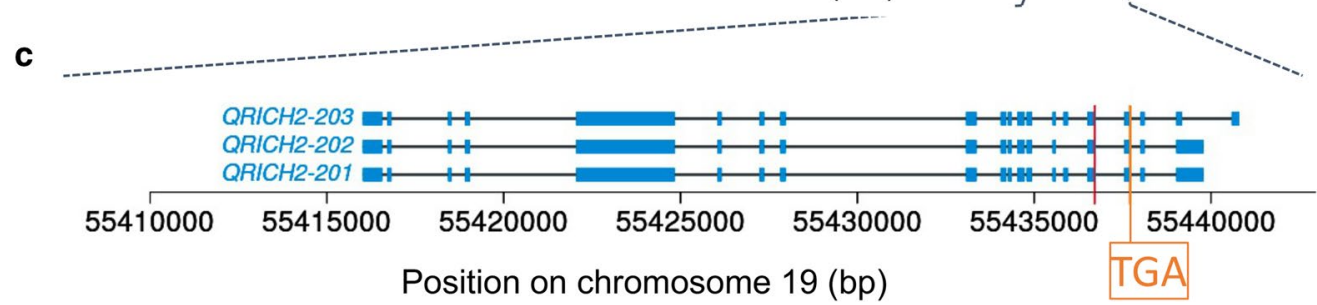

Fig. 1 A 1-bp deletion is a candidate causal variant for a sperm morphology defect in Brown Swiss bulls. a Pedigree of two bulls with a defect of sperm heads and tails. The pedigree only contains suspected carriers of the 1-bp deletion. Ovals and rectangles represent cows and bulls, respectively. The bull born in 2020 and the SAMEA6272098 bull are related through a common ancestor. SAMEA5159783 is the oldest sequenced mutation carrier in the pedigree. $\mathbf{b}$ Each symbol represents the proportion of heterozygous genotypes observed within a 125-kb window for SAMEA6272098. Blue colour represents a 3.625-Mb segment of extended homozygosity encompassing BTA19:55436705TC>T (red vertical line). The green rectangle indicates the position of a BovineHD-based haplotype that encompasses the 1-bp deletion. c Structure of bovine QRICH2 isoforms with transcript-ID ENSBTAT00000065208, ENSBTAT00000064147, and ENSBTAT00000018337 encoding proteins with 1968 (ENSBTAP00000055962), 1934 (ENSBTAP00000054965) and 1827 (ENSBTAP00000018337) amino acids. Blue rectangles represent exons. The red vertical line indicates the position of the 1-bp deletion (BTA19:55436705TC>T). Orange colour indicates the position of a premature termination codon ("TGA") that is introduced due to the shift in translation caused by the 1-bp deletion 
proportion of 34\% Brown Swiss ancestry. In the 1000 Bull Genomes dataset, only SAMEA6272098 carried the deletion in the homozygous state.

QRICH2 mRNA was less abundant for six heterozygous carriers of the 1-bp deletion than for 70 bulls that were homozygous for the wild-type allele $(23.29 \pm 6.76$ vs. $32.13 \pm 7.01$ TPM, P=0.008, Wilcoxon test). Differential expression between heterozygous and wild-type bulls was evident $(\mathrm{P}<0.05$, Wilcoxon test) for 18 QRICH 2 exons (see Additional file 5: Fig. S1). We observed allelic imbalance at the 1-bp deletion in the total RNA sequencing alignments of all six heterozygous bulls; an average of $292 \pm 143$ sequencing reads overlapped with the BTA19:55436705TC $>$ T position, but less than a third $(81 \pm 32)$ supported the deletion. Across all the heterozygous bulls, 1266 and 487 mapped reads supported the reference and alternate allele, respectively $\left(\mathrm{P}=9.45 \times 10^{-80}\right.$, binomial test (see Additional file 5: Fig. S1)), indicating that the RNA of heterozygous bulls is depleted for the QRICH2 transcript carrying the deletion.

\section{Identification of homozygous haplotype carriers in the Brown Swiss population}

To validate the hypothesis that the sperm defect was caused by the 1-bp deletion, we examined ejaculates from homozygous bulls. Because SAMEA6272098 had been slaughtered in 2005 and no semen samples had been preserved, it was not possible to re-examine its sperm defect. In order to identify homozygous carriers for this mutation, we used a dataset that contained 25,243 Brown Swiss and 5228 Original Braunvieh cattle with phased Illumina BovineHD Bead chip genotypes at 17,579 SNPs located on BTA19. These animals were genotyped for routine genomic breeding value prediction in Switzerland. Of the 30,471 genotyped Brown Swiss and Original Braunvieh cattle, 285 (28 females, 257 males) also had whole-genome sequence-called genotypes at the BTA19:55436705TC $>\mathrm{T}$ variant. Twenty-one sequenced animals ( 1 female, 20 males) were heterozygous carriers of the 1-bp deletion. Under the assumption that these 21 animals inherited the 1-bp deletion from a common ancestor, we screened their phased array-derived genotypes for a shared haplotype encompassing BTA19:55436705TC $>\mathrm{T}$. This approach identified a 675-kb haplotype that contained 181 Illumina BovineHD BeadChip SNPs and spanned from 54,992,461 to $55,667,539$ bp on BTA19 and that was present in the heterozygous state in the 21 heterozygous animals (Fig. 1 and see Additional file 2: Table S2).

Of the 264 sequenced animals that did not carry the 1-bp deletion, only one animal (SAMEA7573647) carried the haplotype in the heterozygous state. This animal was a fertile paternal half-sib of SAMEA6272098. Upon manual inspection of the read alignments, we noticed that only one properly mapped sequencing read overlapped with BTA19:55436705TC $>\mathrm{T}$ in the sequence of the SAMEA7573647 bull (see Additional file 6: Fig S2). This read supported the 1-bp deletion, although SAMEA7573647 was genotyped as homozygous for the reference cytosine residue. Low-sequencing coverage likely resulted in undercalling of heterozygous genotypes. Thus, the microarray-derived haplotype was in full concordance with the genotypes at the BTA19:55436705 $\mathrm{TC}>\mathrm{T}$ variant in the 285 sequenced animals.

The $675-\mathrm{kb}$ haplotype had a frequency of $5.4 \%$ among 25,243 genotyped Brown Swiss cattle. This haplotype frequency does not appear to have changed notably over the past 30 years. We detected 2712 heterozygous and 54 homozygous haplotype carriers, whereas 79 homozygous animals were expected assuming random mating, thus the distribution of the observed haplotypes deviated slightly from the Hardy-Weinberg proportions $(P=0.03)$. The maternal grandsire from the heterozygous bull from the Nordic Red Dairy Cattle breed carried the haplotype in the heterozygous state. Among the homozygous haplotype carriers, 35 were females and 19 were males. Since none of the homozygous bulls had been selected as an artificial insemination sire, their semen quality was not examined. Thirty-three of the 35 homozygous females gave birth to at least one offspring, indicating that their fertility was not compromised. Inspection of uncorrected milk records in heterozygous cows indicated that their milk production is normal. None of the 5228 Original Braunvieh cattle carried the 675-kb haplotype.

We used semen quality data from 1142 Brown Swiss bulls that were previously analyzed by Hiltpold et al. [1, 4] to investigate if the 675-kb haplotype is associated with semen quality in the heterozygous state. We examined an average number of 39 (between 5 and 186) and 41 (between 1 and 549) ejaculates from 143 heterozygous haplotype carriers and 999 bulls that did not carry the haplotype, respectively. The absolute values for ejaculate volume (in $\mathrm{mL}$ ), sperm concentration (million sperm per $\mathrm{mL}$ ), and percentage of motile sperm were within reference ranges for both heterozygous carriers $(3.99 \pm 1.16$, $1270 \pm 395$, and $83 \pm 11$ ) and non-carriers of the haplotype $(4.22 \pm 1.21,1234 \pm 328$, and $83 \pm 9)$. The differences in semen quality between carriers and non-carriers of the haplotype were only statistically significant for ejaculate volume ( $\mathrm{P}=0.017$, Wilcoxon test). However, mixed model-based association testing between semen quality and dense markers did not reveal any QTL for semen quality in this genomic region [32]. Thus, we assume that the observed difference in ejaculate is due to factors other than the 1-bp deletion. The proportion of ejaculates that were rejected or contained an excess of sperm with head 
and tail anomalies did not differ notably between heterozygous carriers and non-carriers, supporting a recessive mode of inheritance.

Since all the male homozygous carriers of the haplotype had been slaughtered before our study was conducted, no ejaculate collection and semen analyses could be performed. In order to identify homozygous bulls for phenotypic investigations, the coordinates of the $675-\mathrm{kb}$ haplotype were sent to the Brown Swiss genetic evaluation centers in Switzerland, Austria and Germany. In 2020, a haplotype screen of the genomic selection reference populations identified a 6-month-old bull that carried the haplotype in the homozygous state. The sire of SAMEA6272098 was in the maternal (6th ancestral generation) and paternal (5th ancestral generation) ancestry of the 6-month-old homozygous haplotype carrier indicating inbreeding due to an obligate carrier of the 1-bp deletion (Fig. 1a). Sanger sequencing confirmed homozygosity for the 1-bp deletion (BTA19:55436705TC $>$ T). The bull was apparently regularly developed and healthy.

\section{Ejaculates of a homozygous bull contain immotile sperm with multiple morphological abnormalities}

We kept the bull that was homozygous for the 1-bp deletion at a research station together with other bulls of similar age. At 14 months, its scrotal circumference was relatively small $(31 \mathrm{~cm})$ indicating delayed puberty. The first ejaculate collected from the bull had a volume of $5 \mathrm{~mL}$, but it contained only $0.03 \times 10^{9}$ sperm per $\mathrm{mL}$ (Table 2). None of the sperm were motile and $97.3 \%$ had tail or head abnormalities. The scrotal circumference increased as the bull grew older ( $34.5 \mathrm{~cm}$ at 20 months) but remained relatively small compared to that of the other bulls. We collected six additional ejaculates from the bull at 16, 17, and 20 months. The ejaculates had an average volume of $4.0 \pm 0.9 \mathrm{~mL}$ and contained $0.2 \pm 0.1 \times 10^{9}$ sperm per $\mathrm{mL}$, confirming a normal volume but an aberrantly low sperm concentration and sperm count. More than $99 \%$ of the sperm had major defects and less than $1 \%$ were motile. Histological examination of the testis after slaughter indicated that Leydig cells, rete testis, and tubuli seminiferi were formed normally and spermatogenesis was detectable.

Microscopic analysis of the semen revealed that almost all the sperm had multiple morphological abnormalities that primarily affected the flagella (Fig. 2 and see Additional file 7: Fig. S3). Morphological abnormalities included shortened, coiled, bent, looped, thickened, doubled and absent tails. Loose tails were not detected. Approximately $20 \%$ of the examined sperm were underdeveloped, had irregularly shaped heads, or nuclear vacuoles. Eosin-nigrosin staining indicated that between 4 and $12 \%$ of the sperm were viable in spite of morphological abnormalities of the head and flagella (Fig. 2c).

Transmission electron microscopy cross-sections of sperm from the affected bull confirmed multiple ultrastructural defects of the flagellum and head (Fig. 3 and see Additional file 8: Fig. S4). The axonemes of most flagella deviated from the typical assembly of nine outer microtubule doublets surrounding the central pair. A variable number of outer microtubule doublets was missing in the principal and end piece region of most of the examined flagella. We also detected sperm with multiple flagellar structures within the same cell membrane, indicating that the flagellum was folded around the head instead of being elongated which is typical for underdeveloped sperm (Additional file 8: Fig. S4). Occasionally, we found axonemes in cross-sections of the principal piece that appeared regularly organized.

\section{Discussion}

In this paper, we describe the detection of a recessive 1-bp deletion inducing a frameshift in the QRICH2 gene in two Brown Swiss bulls producing ejaculates with low sperm concentration and immotile sperm cells with multiple morphological abnormalities. Semen samples

Table 2 Semen analysis in a bull homozygous for the 1-bp deletion in the coding sequence of QRICH2

\begin{tabular}{|c|c|c|c|c|c|c|}
\hline $\begin{array}{l}\text { Age of the bull } \\
\text { (months) }\end{array}$ & $\begin{array}{l}\text { Scrotal } \\
\text { circumference }(\mathrm{cm})\end{array}$ & $\begin{array}{l}\text { Ejaculate volume } \\
(\mathrm{mL})\end{array}$ & $\begin{array}{l}\text { Sperm concentration }\left(10^{9}\right. \\
\text { sperm per } \mathrm{mL})\end{array}$ & $\begin{array}{l}\text { Sperm with major } \\
\text { defects (\%) }\end{array}$ & $\begin{array}{l}\text { Motile sperm } \\
\text { (\%) }\end{array}$ & $\begin{array}{l}\text { Viable } \\
\text { sperm } \\
(\%)\end{array}$ \\
\hline 14 & 31 & 5.0 & 0.031 & 97.3 & 0 & na \\
\hline \multirow[t]{2}{*}{16} & 32.5 & 4.0 & 0.187 & 99.6 & $<1$ & na \\
\hline & & 5.0 & 0.115 & 97.9 & $<1$ & na \\
\hline \multirow[t]{2}{*}{17} & 33.5 & 2.8 & 0.160 & 100 & 0 & 12 \\
\hline & & 3.0 & 0.088 & 98.7 & $<1$ & 10.8 \\
\hline \multirow[t]{2}{*}{20} & 34.5 & 4.5 & 0.214 & 99.2 & 0 & 4.2 \\
\hline & & 4.5 & 0.360 & 99.3 & $<1$ & 10.4 \\
\hline
\end{tabular}



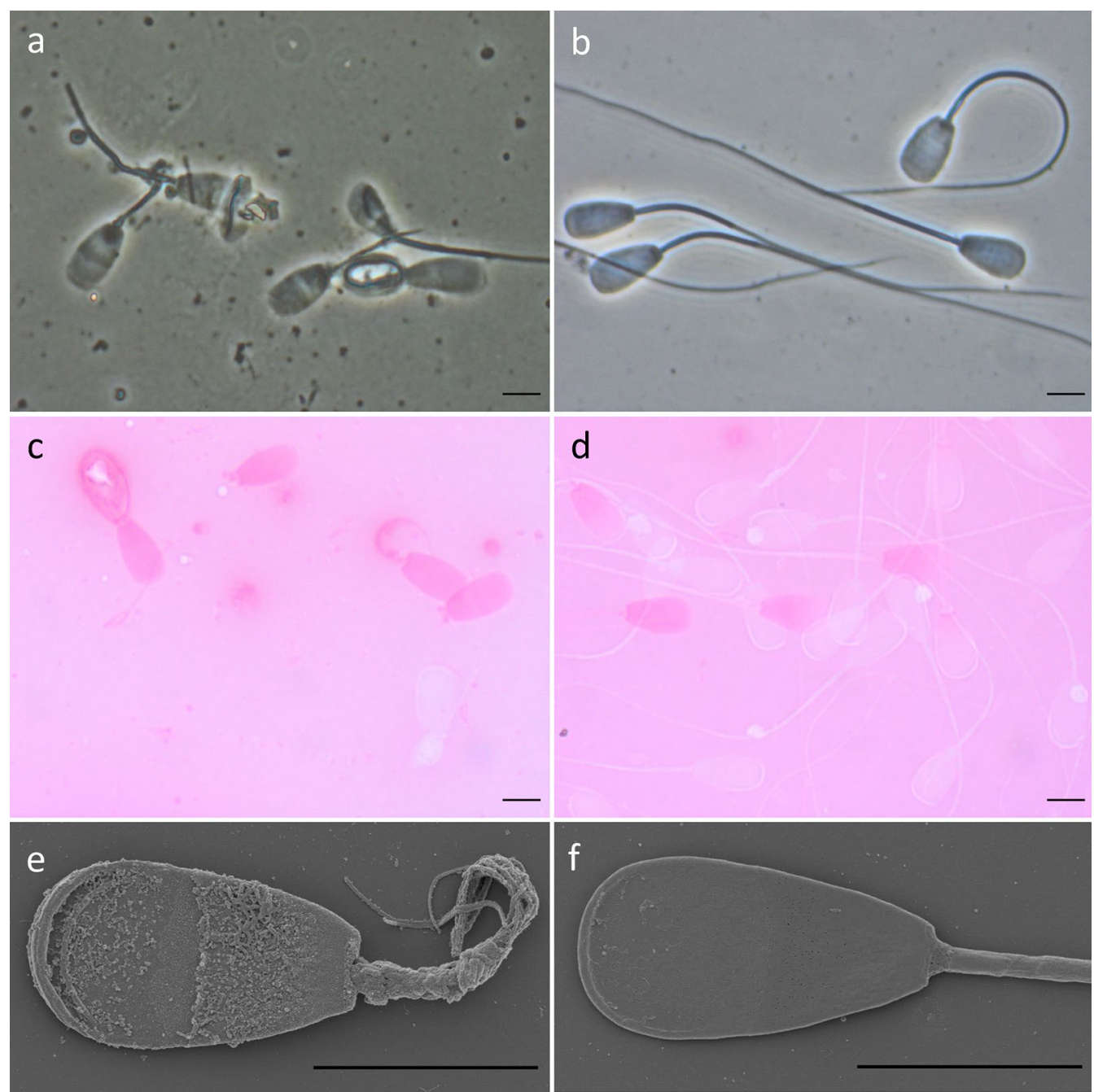

Fig. 2 Sperm of a bull homozygous for the 1-bp deletion and of a control bull. Representative phase-contrast (a, b), eosin-nigrosin stained (c, d), and scanning electron microscopy images (e, $\mathbf{f})$ of sperm of a bull homozygous for the 1-bp deletion $(\mathbf{a}, \mathbf{c}, \mathbf{e})$ and of a control bull (b, $\mathbf{d}, \mathbf{f})$ with normal sperm quality. Sperm of the affected bull show multiple morphological abnormalities of head and flagella (a). Phase-contrast microscopy also revealed numerous cell debris particles. Viable sperm remain unstained and appear white in eosin-nigrosin stained images whereas dead sperm are stained and appear purple $(\mathbf{c}, \mathbf{d})$. Flagella of the affected bull are irregularly shaped and mostly have an uneven surface (e). Scale bar: $5 \mu \mathrm{m}$

from these bulls were not used for artificial inseminations because the resulting disorder very likely prevents fertilization in vivo due to the inability of the sperm to move. Intracytoplasmic sperm injection (ICSI) although not a common practice in cattle breedingcould possibly enable fertilization because some sperm were viable and had normally shaped heads. In humans, fertilization and normal offspring development were obtained with ICSI using sperm with multiple morphological abnormalities of the flagella that were caused by deleterious variants in QRICH2 [36].
We applied an indirect haplotype test and found that the 1-bp deletion segregates at a frequency of $5 \%$ in the Brown Swiss population. Assuming random mating, the deletion is expected to occur in the homozygous state in 1 out of 400 bulls. However, the proportion of homozygous bulls was lower in our study. None of the 1142 Brown Swiss bulls for which we had microarray-derived genotypes and routinely assessed semen quality phenotypes, carried the haplotype in the homozygous state. The 1-bp deletion resides in a haplotype that is inherited from a common ancestor. The mating of closely related 


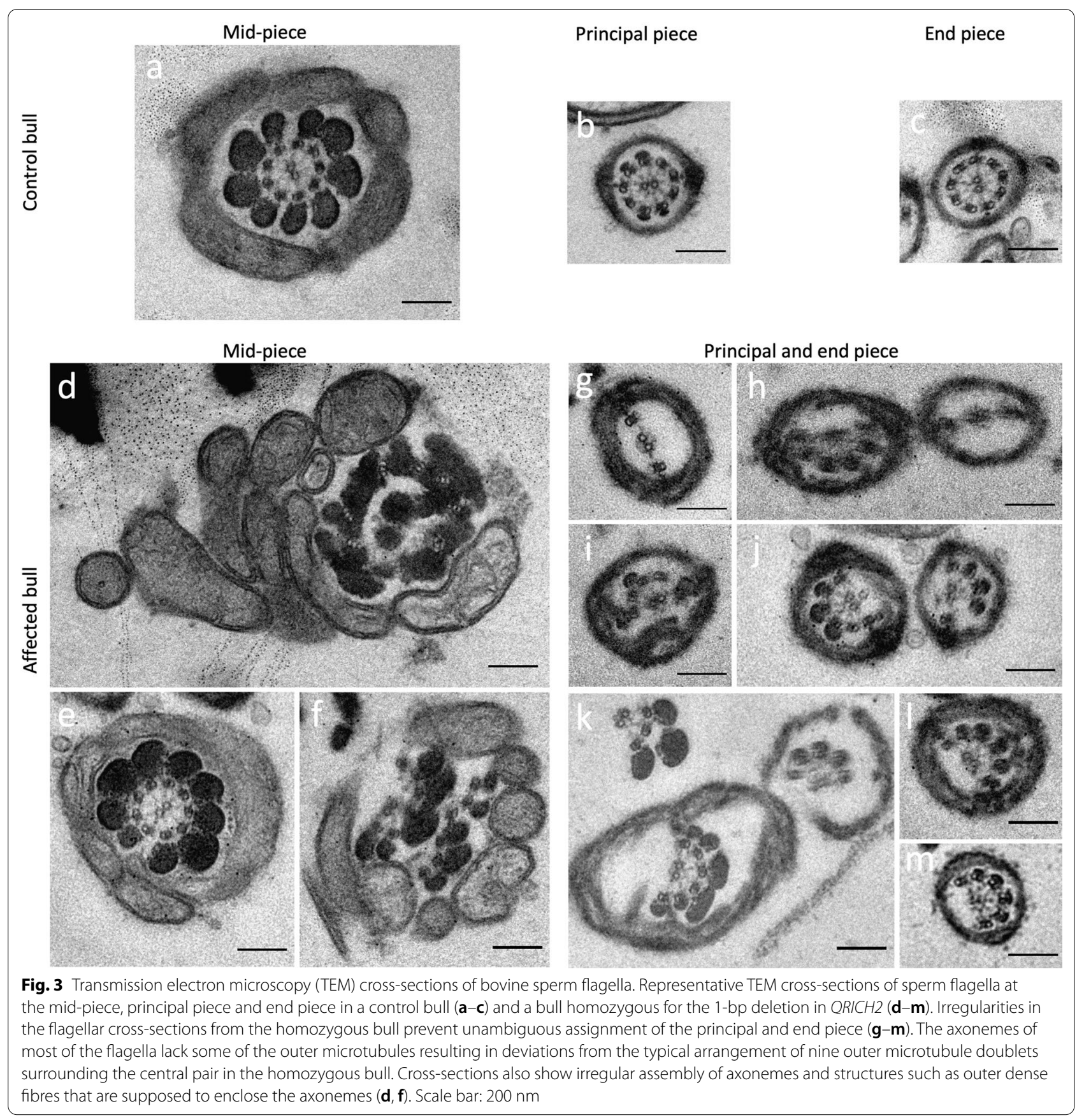

individuals is generally avoided to prevent inbreeding and therefore haplotypes may occur less frequently in the homozygous state than expected under random mating. In principle, the frequency of the 1-bp deletion may be lower than estimated from an indirect haplotype test. If a mutation occurred relatively recently, the haplotype encompassing the mutation may be indistinguishable from its ancestral form that does not carry the mutation. In such a situation, a haplotype-based test overestimates the frequency of the mutation [37]. However, we found no evidence that an ancestral version of the haplotype segregates in the Brown Swiss population, as the 675-kb haplotype was in full concordance with the 1-bp deletion. Moreover, the frequency of this haplotype agreed well with the frequency of the 1-bp deletion estimated from whole-genome sequence variants.

The birth years of the two homozygous bulls span almost two decades. Only one of these bulls was selected 
as a potential artificial insemination sire. Since immotile sperm with multiple morphological abnormalities were detected during routine semen analyses, all ejaculates from this bull were discarded and not used for artificial inseminations. Heterozygous bulls produce normal sperm. If the semen of prospective artificial insemination and natural mating bulls is examined, this sperm defect is easily recognized and thus will not manifest itself in repeated breedings and low non-return rates as is the case for infertility disorders that are not detected from standard semen analyses [8]. Economic losses due to this defect are negligible in the Brown Swiss cattle population because the frequency of the 1-bp deletion is low and aberrant semen quality of homozygous bulls can be easily detected.

The widespread use of individual carriers of this mutation in artificial insemination can result in the occurrence of frequent recessive alleles within a short time [38]. It is advised to monitor the BTA19:55436705TC $>\mathrm{T}$ variant in the Brown Swiss population, e.g., by using customized microarrays, and to implement genome-based mating programs to avoid the frequent emergence of bulls that produce ejaculates containing immotile sperm. We did not detect the BTA19:55436705TC $>$ T variant in Swiss Original Braunvieh animals, which suggests that the mutation occurred relatively recently possibly after the separation of the Braunvieh breed into the dairy (Brown Swiss) and dual-purpose (Original Braunvieh) lineages. We found the 1-bp deletion in one animal from the Nordic Red Dairy Cattle breed, which likely resulted from crossbreeding with a heterozygous Brown Swiss sire [39]. Thus, our results increase the number of known alleles with undesired effects that have been introgressed from foreign breeds into the Nordic Red Dairy Cattle breed [40-42].

Human and mouse orthologs of the QRICH2 gene harbour loss-of-function variants that result in multiple morphological abnormalities and ultrastructural defects of the sperm flagella, thereby leading to immotile sperm and infertility in vivo [34, 35, 43]. We have replicated these results by analyzing the ejaculates from two bulls that are homozygous for a frameshift-inducing 1-bp deletion in the bovine QRICH2 gene, which suggests an evolutionarily conserved protein function. The 1-bp deletion is predicted to induce a premature termination codon that shortens the protein by 131 amino acids. The truncated protein may be retained with reduced functionality or the transcript may be degraded via nonsense-mediated mRNA decay. The position of the premature termination codon complies with both canonical and non-canonical rules for nonsense-mediated mRNA decay [44-46]. Allelic imbalance at the 1-bp deletion and the lower levels of QRICH2 mRNA in the testis transcriptomes of heterozygous than in those of wild-type bulls confirm that the premature termination codon triggers nonsensemediated mRNA decay. These findings show that the 1-bp deletion constitutes a loss-of-function allele of the bovine QRICH2 gene, thereby supporting the pathogenicity of a predicted deleterious variant that resides in a similar domain of the human QRICH2 gene [35].

To date, the QRICH2 protein has primarily been implicated in flagellar formation of the sperm. Apart from flagellar abnormalities, we observed sperm head anomalies, drastically reduced sperm concentration and low sperm count in all the ejaculates examined from bulls homozygous for the 1-bp deletion. Sperm concentration and total sperm count were also at the lower bound of the normal range in humans with deleterious QRICH2 alleles [34, 35, 43]. These findings suggest that loss of QRICH2 functionality not only compromises the assembly of sperm flagella, but generally impairs spermiogenesis. Variants other than the 1-bp deletion may contribute to the aberrant semen quality of the two examined Brown Swiss bulls. However, pinpointing putative modifier loci is difficult for rare alleles [47] and was not attempted for the 1-bp deletion in QRICH2.

To the best of our knowledge, this is the first time that an effect arising from a loss-of-function allele in QRICH2 is described in a species other than mice and humans. Apart from producing anomalous ejaculates, homozygous bulls are indistinguishable from wild-type and heterozygous individuals. Homozygous cows were not examined clinically in our study, but their normal fertility and milk production likely indicate an overall normal health [48]. Moreover, no QTL for economically relevant traits have been detected next to BTA19:55436705TC $>$ T in Brown Swiss cattle [49]. These findings suggest that the 1-bp deletion does not compromise traits other than semen quality. This agrees with loss-of-function alleles in other genes with extreme testis-biased expression [8-10, $50]$ and corroborates findings in humans and mice with loss-of-function alleles in QRICH2 [34], which suggest that QRICH2 is not essential for somatic development in mammals. The deviation from Hardy-Weinberg proportions $(\mathrm{P}=0.03)$ of the haplotype encompassing the 1-bp deletion is likely due to either selective breeding or the inability of homozygous males to contribute to the next generation rather than to fatal consequences arising from homozygosity.

Our analyses in two homozygous bulls provide evidence for the causality of the 1-bp deletion from a statistical, functional, and comparative genomics point of view $[51,52]$. We discovered the deletion using a phenotypedriven approach in a historic animal sample and verified its phenotypic consequences in a second bull that was born almost two decades later and was identified through 
a genotype-driven screen. Transcriptome analyses show that the 1-bp deletion triggers nonsense-mediated mRNA decay, which confirms that it constitutes a loss-of-function allele. Orthologs of the bovine QRICH2 gene harbour loss-of-function alleles; some of them reside in the same domain as the 1-bp deletion identified in our study and cause sperm defects mirroring those of the homozygous Brown Swiss bulls, which suggests that QRICH2 is essential for male fertility in mammals. Although a formal proof for the causality remains to be produced, these pieces of evidence are sufficient to warrant the monitoring of the 1-bp deletion in cattle breeding programs.

\section{Conclusions}

A 1-bp deletion in the coding sequence of the bovine QRICH2 gene is likely a causal mutation for low sperm concentration and immotile sperm with multiple morphological abnormalities in the homozygous state. The 1-bp deletion has a frequency of 5\% in the Brown Swiss cattle population. Homozygous bulls are unsuitable for breeding as they are very likely infertile in vivo. Apart from having a poor semen quality, these homozygous bulls are indistinguishable from heterozygous and wildtype animals. Although the immediate economic consequences due to the undesired allele are negligible, the monitoring of the defective allele in the Brown Swiss cattle population using customized genotyping is recommended to avoid increasing its frequency.

\section{Supplementary Information}

The online version contains supplementary material available at https://doi. org/10.1186/s12711-022-00710-0.

Additional file 1: Table S1. Sequence read archive accession numbers for the sequenced animals. The spreadsheet file contains accession numbers from the European Nucleotide Archive that point to sequencing data for cattle from various breeds. The data in the file are organized in three spreadsheets. The accession for the bull that produced ejaculates with poor quality is listed in «case_SRA_ID». Accessions for 397 fertile control animals from various breeds are listed in «controls_SRA_IDs». Accessions, sequence-based genotypes and haplotypes for 285 cattle used to assign the 1-bp deletion onto a haplotype are listed in «array-based genotypes».

Additional file 2: Table S2. Coordinates of a diagnostic haplotype. Illumina BovineHD coordinates (SNP-name, chromosome, physical position [ARS-UCD1.2]) and haplotype allele of 181 markers that define the diagnostic 675-kb haplotype.

Additional file 3: Table S3. Accessions for the eQTL cohort. Accessions for sequencing reads obtained from RNA and DNA prepared from testis tissue of 76 mature bulls.

Additional file 4: Table S4. Candidate causal variants. Functional consequences predicted for 1655 variants that are compatible with recessive inheritance based on the Ensembl (version 104) and Refseq (version 106) annotation of the bovine genome.

Additional file 5: Figure S1. QR/CH2 mRNA analysis. (a) Exon-specific expression (quantified in transcripts per million [TPM]) for QRICH2 in the testis tissue of six heterozygous (green) and 70 homozygous wild-type (grey) bulls. Red asterisks indicate exons that were differentially expressed
( $P<0.05$, Wilcoxon rank sum test) between heterozygous carriers and wild type bulls. (b) Integrative Genomics Viewer coverage tracks from RNA sequence read alignments overlapping the frameshift-inducing 1-bp deletion (red arrow) in six heterozygous bulls. The identifiers of the coverage tracks refer to accessions from the sequence read archive.

Additional file 6: Figure S2. DNA sequence alignment of SAMEA6272098. Output from «samtools tview» centered on BTA19:55436705TC>T representing DNA sequence read alignments from a bull (SAMEA6272098) that carries the 675-kb haplotype in the heterozygous state, but was genotyped as homozygous for the reference allele at the position of the BTA19:55436705TC>T variant. The asterisk within the only read that overlaps BTA19:55436706 indicates that the bull carries the 1-bp deletion.

Additional file 7: Figure S3. Phase-contrast images of sperm from a bull homozygous for the 1-bp deletion. All images display sperm with major sperm head and/or flagellar defects. Very short, shortened or absent flagella were the most prevalent flagellar abnormalities: very short flagella $(a, b, d, e, f, g, i, k, s, u)$, shortened flagella (c, e, h, i, l, $q, r$, , ) and absent flagella $(c, i, j, o, t)$. Short doubled $(e, k, s)$ or short thickened $(e, k, i, n, q)$ were apparent too. Some flagella were strongly folded ( $m$, y right sperm) or coiled (y left sperm). Many sperm showed defective heads as well as defective flagella: pyriform $(c, i, m, s, t)$, round $(g, r)$, abnormal contour $(p)$, and diadem defect/vacuoles $(b, o, q, u, y)$. Underdeveloped sperm with the flagella strongly folded around the sperm head are considered the most severe sperm defects in bull ( $d$ right sperm, t right sperm, $v, w, x$ ). Scale bar: $5 \mu \mathrm{m}$.

Additional file 8: Figure S4. TEM and SEM images of sperm from a bull homozygous for the 1-bp deletion. TEM cross-section of an underdeveloped sperm with multiple flagellar structures next to the nucleus (black) enclosed by a cell membrane (a). Underdeveloped sperm with the flagellum curled on the head visualized using SEM (b). Longitudinal TEM cross-section (c) and SEM (d) of sperms with a thickened vesicularized structure at the sperm neck and multiple disorganized flagellar structures at the mid-piece. Longitudinal TEM cross-section of the neck region of a normal sperm from a control bull (e). Scale bar: $1 \mu \mathrm{m}$.

\section{Acknowledgements}

We thank Dr. Franz Birkenmaier (Allgäuer Herdbuchgesellschaft) for support in identifying homozygous bulls and Dr. Melissa Terranova and Flavio Ferrari (AgroVet-Strickhof) for the handling of the bull homozygous for the 1-bp deletion. We thank Braunvieh Schweiz for providing genotype data. We are thankful for the excellent technical support provided by the ETH Zürich technology platform FGCZ (https://fgcz.ch/) concerning DNA and RNA sequencing. We are grateful to Dr. Cecilia Bebeacua and Stephan Handschin from the ETH Zürich technology platform ScopeM (https://scopem.ethz.ch/) for outstanding support in electron microscopy. We appreciate support from veterinarians from the meat inspection and the slaughterhouse staff during the sampling of bull testes as well as Till Graf, Patrick Fitzi, and Remo Hengartner for their help in collecting tissue.

\section{Authors' contributions}

HP conceived the study. MH and HP analysed the data. MH and FJ analysed semen samples of the homozygous bull. NKK called sequence variant genotypes. XMM established the eQTL cohort. ZHF carried out sequencing experiments. HP, HS and FRS analysed genotyping data from the Brown Swiss reference populations. MS organised genetic material for sequencing. UW provided semen quality data. HP and MH wrote the manuscript. All authors read and approved the final manuscript.

\section{Funding}

Open access funding provided by Swiss Federal Institute of Technology Zurich. This study received financial support from Swissgenetics, Zollikofen, Switzerland (https://swissgenetics.ch/). The sequencing of Brown Swiss animals was supported by a grant from the Swiss National Science Foundation (310030 185229). The establishment of an eQTL cohort was supported by an ETH Zurich Research Grant and Swissgenetics. 


\section{Data availability}

Whole-genome sequencing data of 397 fertile bulls and the infertile bull are available at the European Nucleotide Archive (ENA) of the EMBL under sample accessions listed in Additional file 1: Table S1. Whole-genome sequencing data of the 285 cattle used to identify the trait-associated haplotype are available at the European Nucleotide Archive (ENA) of the EMBL under sample accessions listed in Additional file 1: Table S1. DNA and RNA sequencing data of the 76 bulls in the eQTL cohort are available at the European Nucleotide Archive (ENA) of the EMBL under sample accessions listed in Additional file 3: Table S3.

\section{Declarations}

\section{Ethics approval and consent to participate}

Our study was approved by the veterinary office of the Canton of Zurich, Switzerland (animal experimentation permit ZH 181/19).

\section{Consent for publication}

Not applicable.

\section{Competing interests}

Ulrich Witschi is an employee of Swissgenetics. All other authors declare that they have no competing interests.

\section{Author details}

${ }^{1}$ Animal Genomics, Institute of Agricultural Sciences, ETH Zürich, Universitätstrasse 2, 8092 Zürich, Switzerland. ${ }^{2}$ Clinic of Reproductive Medicine, Vetsuisse Faculty, University of Zürich, Winterthurerstrasse 260, 8057 Zürich, Switzerland. ${ }^{3}$ ZuchtData EDV Dienstleistungen, Dresdner Straße 89/B1/18, 1200 Wien, Austria. ${ }^{4}$ Qualitas AG, Chamerstrasse 56, 6300 Zug, Switzerland. ${ }^{5}$ Swissgenetics, Meielenfeldweg 12, 3052 Zollikofen, Switzerland. ${ }^{6}$ Present Address: Genome Biology of Neurodegenerative Diseases, Deutsches Zentrum Für Neurodegenerative Erkrankungen e. V. (DZNE), Otfried-Müller-Str. 23, 72076 Tübingen, Germany.

Received: 19 November 2021 Accepted: 17 February 2022 Published online: 07 March 2022

\section{References}

1. Hiltpold M, Niu G, Kadri NK, Crysnanto D, Fang ZH, Spengeler M, et al. Activation of cryptic splicing in bovine WDR19 is associated with reduced semen quality and male fertility. PLoS Genet. 2020;16:e1008804.

2. Schaeffer LR. Evaluation of bulls for nonreturn rates within artificial insemination organizations. J Dairy Sci. 1993;76:837-42.

3. Taylor JF, Schnabel RD, Sutovsky P. Review: Genomics of bull fertility. Animal. 2018;12:s172-83.

4. Hiltpold M, Kadri NK, Janett F, Witschi U, Schmitz-Hsu F, Pausch H. Autosomal recessive loci contribute significantly to quantitative variation of male fertility in a dairy cattle population. BMC Genomics. 2021;22:225.

5. Nani JP, Peñagaricano F. Whole-genome homozygosity mapping reveals candidate regions affecting bull fertility in US Holstein cattle. BMC Genomics. 2020:21:338.

6. Ferenčaković M, Sölkner J, Kapš M, Curik I. Genome-wide mapping and estimation of inbreeding depression of semen quality traits in a cattle population. J Dairy Sci. 2017;100:4721-30.

7. Mathevon M, Buhr MM, Dekkers JC. Environmental, management, and genetic factors affecting semen production in Holstein bulls. J Dairy Sci. 1998:81:3321-30.

8. Pausch H, Kölle S, Wurmser C, Schwarzenbacher H, Emmerling R, Jansen $\mathrm{S}$, et al. A nonsense mutation in TMEM95 encoding a nondescript transmembrane protein causes idiopathic male subfertility in cattle. PLoS Genet. 2014;10:e1004044.

9. Pausch H, Venhoranta H, Wurmser C, Hakala K, Iso-Touru T, Sironen A, et al. A frameshift mutation in ARMC3 is associated with a tail stump sperm defect in Swedish Red (Bos taurus) cattle. BMC Genet. 2016;17:49.

10. Iso-Touru T, Wurmser C, Venhoranta H, Hiltpold M, Savolainen T, Sironen A, et al. A splice donor variant in CCDC189 is associated with asthenospermia in Nordic Red dairy cattle. BMC Genomics. 2019;20:286.
11. Abdollahi-Arpanahi R, Pacheco HA, Peñagaricano F. Targeted sequencing reveals candidate causal variants for dairy bull subfertility. Anim Genet. 2021:52:509-13.

12. Chen S, Zhou Y, Chen Y, Gu J. Fastp: an ultra-fast all-in-one FASTQ preprocessor. Bioinformatics. 2018;34:1884-90.

13. Li H. Aligning sequence reads, clone sequences and assembly contigs with BWA-MEM. arXiv:13033997. 2013.

14. Tarasov A, Vilella AJ, Cuppen E, Nijman IJ, Prins P. Sambamba: fast processing of NGS alignment formats. Bioinformatics. 2015;31:2032-4.

15. Pedersen BS, Quinlan AR. Mosdepth: quick coverage calculation for genomes and exomes. Bioinformatics. 2018;34:867-8.

16. DePristo MA, Banks E, Poplin RE, Garimella KV, Maguire JR, Hartl C, et al. A framework for variation discovery and genotyping using next-generation DNA sequencing data. Nat Genet. 2011;43:491-8.

17. Browning BL, Browning SR. A unified approach to genotype imputation and haplotype-phase inference for large data sets of trios and unrelated individuals. Am J Hum Genet. 2009;84:210-23.

18. Crysnanto $D$, Wurmser $C$, Pausch $H$. Accurate sequence variant genotyping in cattle using variation-aware genome graphs. Genet Sel Evol. 2019;51:21.

19. McLaren W, Gil L, Hunt SE, Riat HS, Ritchie GRS, Thormann A, et al. The Ensembl variant effect predictor. Genome Biol. 2016;17:122.

20. Browning BL, Browning SR. Genotype imputation with millions of reference samples. Am J Hum Genet. 2016;98:116-26.

21. Kadri NK, Mapel XM, Pausch $H$. The intronic branch point sequence is under strong evolutionary constraint in the bovine and human genome. Commun Biol. 2021;4:1206.

22. Rosen BD, Bickhart DM, Schnabel RD, Koren S, Elsik CG, Tseng E, et al. De novo assembly of the cattle reference genome with single-molecule sequencing. Gigascience. 2020;9:giaa021.

23. Dobin A, Davis CA, Schlesinger F, Drenkow J, Zaleski C, Jha S, et al STAR: ultrafast universal RNA-seq aligner. Bioinformatics. 2013;29:15-21.

24. Bray NL, Pimentel H, Melsted P, Pachter L. Near-optimal probabilistic RNA-seq quantification. Nat Biotechnol. 2016;34:525-7.

25. Soneson C, Love MI, Robinson MD. Differential analyses for RNA-seq: transcript-level estimates improve gene-level inferences. F1000Res. 2015:4:1521.

26. Delaneau O, Ongen H, Brown AA, Fort A, Panousis NI, Dermitzakis ET. A complete tool set for molecular QTL discovery and analysis. Nat Commun. 2017;8:15452.

27. Garrido-Martín D, Palumbo E, Guigó R, Breschi A. ggsashimi: Sashimi plot revised for browser- and annotation-independent splicing visualization. PLoS Comput Biol. 2018;14:e1006360.

28. Robinson JT, Thorvaldsdóttir H, Winckler W, Guttman M, Lander ES, Getz G, et al. Integrative genomics viewer. Nat Biotechnol. 2011;29:24-6.

29. Hancock JL. The morphology of boar spermatozoa. J R Microsc Soc. 1956;76:84-97.

30. Blom E. The ultrastructure of some characteristic sperm defects and a proposal for a new classification of the bull spermiogram (author's transl). Nord Vet Med. 1973;25:383-91.

31. Blom E. A one-minute live-dead sperm stain by means of eosinnigrosin. Fertil Steril. 1950;1:176-7.

32. Mapel XM, Hiltpold M, Kadri NK, Witschi U, Pausch H. Bull fertility and semen quality are not correlated with dairy and production traits in Brown Swiss cattle. JDS Commun. 2022. (in press).

33. Fang L, Cai W, Liu S, Canela-Xandri O, Gao Y, Jiang J, et al. Comprehensive analyses of 723 transcriptomes enhance genetic and biological interpretations for complex traits in cattle. Genome Res. 2020;30:790-801.

34. Shen $Y$, Zhang F, Li F, Jiang $X$, Yang $Y$, Li X, et al. Loss-of-function mutations in QR/CH2 cause male infertility with multiple morphological abnormalities of the sperm flagella. Nat Commun. 2019;10:433.

35. Kherraf Z-E, Cazin C, Coutton C, Amiri-Yekta A, Martinez G, Boguenet M, et al. Whole exome sequencing of men with multiple morphological abnormalities of the sperm flagella reveals novel homozygous QRICH2 mutations. Clin Genet. 2019;96:394-401.

36. Xu W, Li XL. Exome sequencing identifying additional QR/CH2 mutations in oligo-astheno-teratozoospermia and asthenospermia patients. Fertil Steril. 2019;112:e376. 
37. Jung S, Pausch H, Langenmayer MC, Schwarzenbacher H, MajzoubAltweck M, Gollnick NS, et al. A nonsense mutation in PLD4 is associated with a zinc deficiency-like syndrome in Fleckvieh cattle. BMC Genomics. 2014;15:623.

38. Schwarzenbacher $\mathrm{H}$, Wurmser $\mathrm{C}$, Flisikowski K, Misurova L, Jung S, Langenmayer MC, et al. A frameshift mutation in GON4L is associated with proportionate dwarfism in Fleckvieh cattle. Genet Sel Evol. 2016;48:25.

39. Zhang Q, Calus MPL, Bosse M, Sahana G, Lund MS, Guldbrandtsen B. Human-mediated introgression of haplotypes in a modern dairy cattle breed. Genetics. 2018;209:1305-17.

40. Pausch $\mathrm{H}$, Ammermüller $\mathrm{S}$, Wurmser $\mathrm{C}$, Hamann $\mathrm{H}$, Tetens J, Drögemüller $C$, et al. A nonsense mutation in the COL7A1 gene causes epidermolysis bullosa in Vorderwald cattle. BMC Genet. 2016;17:149.

41. Schwarzenbacher H, Burgstaller J, Seefried FR, Wurmser C, Hilbe M, Jung $\mathrm{S}$, et al. A missense mutation in TUBD1 is associated with high juvenile mortality in Braunvieh and Fleckvieh cattle. BMC Genomics. 2016;17:400.

42. Wu X, Mesbah-Uddin M, Guldbrandtsen B, Lund MS, Sahana G. Novel haplotypes responsible for prenatal death in Nordic Red and Danish Jersey cattle. J Dairy Sci. 2020;103:4570-8.

43. Oud MS, Houston BJ, Volozonoka L, Mastrorosa FK, Holt GS, Alobaidi $B K S$, et al. Exome sequencing reveals variants in known and novel candidate genes for severe sperm motility disorders. Hum Reprod. 2021;36:2597-611.

44. Brogna S, Wen J. Nonsense-mediated mRNA decay (NMD) mechanisms. Nat Struct Mol Biol. 2009;16:107-13.

45. Lindeboom RGH, Vermeulen M, Lehner B, Supek F. The impact of nonsense-mediated mRNA decay on genetic disease, gene editing and cancer immunotherapy. Nat Genet. 2019;51:1645-51.

46. Teran NA, Nachun DC, Eulalio T, Ferraro NM, Smail C, Rivas MA, et al. Nonsense-mediated decay is highly stable across individuals and tissues. Am J Hum Genet. 2021;108:1401-8.

47. Bourneuf E, Otz P, Pausch H, Jagannathan V, Michot P, Grohs C, et al. Rapid discovery of de novo deleterious mutations in cattle enhances the value of livestock as model species. Sci Rep. 2017;7:11466.

48. Reynolds EGM, Neeley C, Lopdell TJ, Keehan M, Dittmer K, Harland CS, et al. Non-additive association analysis using proxy phenotypes identifies novel cattle syndromes. Nat Genet. 2021;53:949-54.

49. Fang Z-H, Pausch $\mathrm{H}$. Multi-trait meta-analyses reveal 25 quantitative trait loci for economically important traits in Brown Swiss cattle. BMC Genomics. 2019;20:695.

50. Nosková A, Wurmser C, Crysnanto D, Sironen A, Uimari P, Fries R, et al. Deletion of porcine $B O L L$ is associated with defective acrosomes and subfertility in Yorkshire boars. Anim Genet. 2020;51:945-9.

51. Sunyaev SR. Inferring causality and functional significance of human coding DNA variants. Hum Mol Genet. 2012;21:R10-7.

52. MacArthur DG, Manolio TA, Dimmock DP, Rehm HL, Shendure J, Abecasis $G R$, et al. Guidelines for investigating causality of sequence variants in human disease. Nature. 2014;508:469-76.

\section{Publisher's Note}

Springer Nature remains neutral with regard to jurisdictional claims in published maps and institutional affiliations.

Ready to submit your research? Choose BMC and benefit from:

- fast, convenient online submission

- thorough peer review by experienced researchers in your field

- rapid publication on acceptance

- support for research data, including large and complex data types

- gold Open Access which fosters wider collaboration and increased citations

- maximum visibility for your research: over $100 \mathrm{M}$ website views per year

At BMC, research is always in progress.

Learn more biomedcentral.com/submissions 